\title{
Téoros
}

Revue de recherche en tourisme

\section{Le tourisme : un moyen de sensibilisation à l'environnement}

\section{Roger Nadeau et François Thomas}

Volume 10, numéro 1, mars 1991

Tourisme et environnement

URI : https://id.erudit.org/iderudit/1079811ar

DOI : https://doi.org/10.7202/1079811ar

Aller au sommaire du numéro

Éditeur(s)

Université du Québec à Montréal

ISSN

0712-8657 (imprimé)

1923-2705 (numérique)

Découvrir la revue

Citer cet article

Nadeau, R. \& Thomas, F. (1991). Le tourisme : un moyen de sensibilisation à

l'environnement. Téoros, 10(1), 3-42. https://doi.org/10.7202/1079811ar d'utilisation que vous pouvez consulter en ligne.

https://apropos.erudit.org/fr/usagers/politique-dutilisation/ 


\section{Le tourisme : \\ un moyen de sensibilisation à l'environnement}

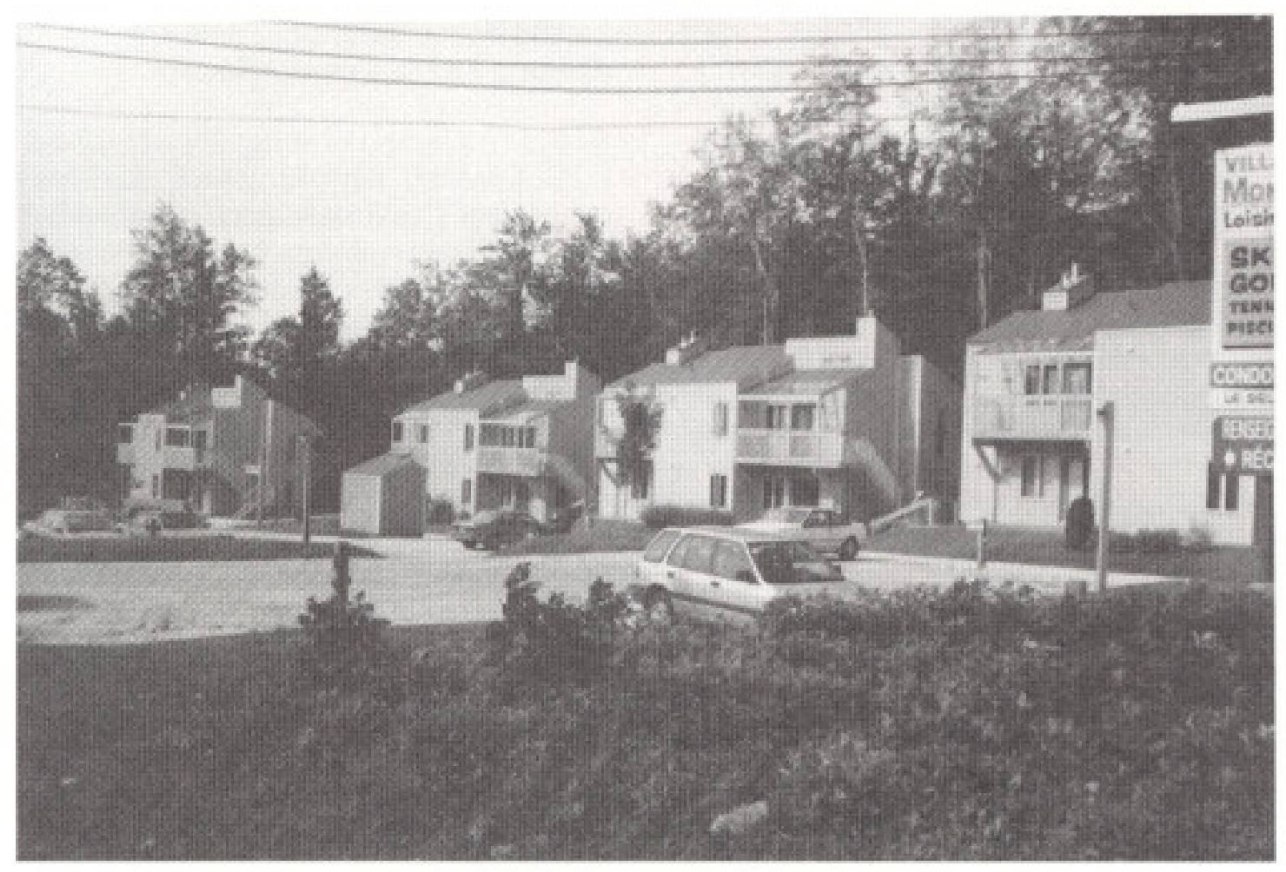

"L'objectiffinal de toute politique touristique consiste à assurer a long terme, à toutes les couches de la population. la détente physique et psychique ainsi que des activités sociales dans un paysage intact, aménagé selon les exigences de l'environnement, les besoins des vacanciers et les intérêts à long terme des populations indigènes." (Jost Krippendorf, $1977)^{(1)}$
Monsieur Foger Nadeau est protesseur de tourisme et directeur du Département de géographie et teledétection de |'Univer sité deSherbrooke. Monsieur François Thomas detient un bacealaureat en urbanisme de l'UOAM et poursuit une maitrise en geographie à l'Uniwersité de Sherbrooke.
La peur ou la sagesse?

'Depuis une décennie surtout, la sensibilisation à l'environnement a fait des progrès enormes, au point que les sondages nous révèlent que cette question est devenue une des toutes premières priorités dans l'échelle de valeur de nos populations. Au début des années 1980, le ministre québécois Marcel Léger décrétait que les 6,5 millions de Québécois étaient promus au rang de "gardiens de l'environnement"; en 1990 , le gouvernement fédéral a déposé son "Plan Vert" de trois milliards de dollars, en faisant un projet de société majeur. Bref, l'environnement, $c$ 'est le sujet de l'heure, c'est la mode in; si tu es "branché", tu es préoccupé d'environnement, de conservation, d'écologie, de pluies acides, de couche d'ozone et de qualité de vie. Si les questions environnementales sont perçues avec une telle acuité, c'est bien sûr parce que l'on a bien compris qu'il s'agit de bien plus que d'une mode, qu'il s'agit en fait d'une question de survie pour cette charmante planete Terre... et pour ses habitants. Il y a encore bien du chemin à faire et il est dommage que ce soit la peur qui engendre la sagesse!

\section{Le tourisme pointé du doigt}

L'industrie touristique du Québec est intimement associée à la problématique environnementale que nous vivons et, cela, pour deux raisons principales et souvent contradictoires: d'une part, l'activité touristique est souvent accusće d'être une source importante de dégradation du milieu naturel et, d'autre part, ce milieu naturel de qualité, constituant la matière premiẹre du tourisme, doit impérativement être sauvegardé sous peine de voir cette industrie majeure $s^{*}$ auto-détruire.

Cen'est pas sans raison que l'on s' inquiète de l'impact que sont susceptibles de générer les projets de développement touristique d'envergure qui naissent etpoussent parfois sans contrôle ni planification, en fonction d'une vision à très court terme. "Sans cracher de fumée, sans déverser de BPC, le tourisme, comme toute industrie, a pourtant un impact immense sur l'environnement: quand ils dé barquent en masse, les touristes causent parfois des ravages qui feraient passer l'Inco ou Union Carbide pour des enfants de choeur. Pour eux, on bulldoze des forêts, on draine des marais, on construit de nouvelles routes, de nouveaux aéroports et même de nouvelles villes! ${ }^{p(2)}$

Toutes les statistiques sont unanimes: le nombre de touristes dans le monde va continuer de croître, ce qui va accentuer la pression sur le milieu naturel. N'est-ce pas Krippendorf qui observait que les vacanciers modernes, c'est un peu "tout le monde pour les mêmes raisons au mểme endroit en même temps"n?̣(3) Et le développement touristique a ceci de particulier qu'il s'installe prioritairement en zones ecologiquement fragiles, très fragiles. Le tourisme s'implante d'abord sur les bords de mer, les rives de lacs et de rivières, les flancs de montagnes, lesquels sont des écosystèmes fragiles qui peuvent se détériorer rapidement. Mais qui sont les responsables de ces écarts commis au nom du tourisme? Est-ce que ce sont les touristes eux-mêmes, des promoteurs - développeurs trop pressés ou des pouvoirs publics complaisants et insouciants? L'industrie touristique jouit-elle d'un traitement particulier? 
Pour plusieurs gens bien pensants, un projet touristique est automatiquement une calamité qu'il faut combattre, une menace épouvantable qu'il faut tuer dans l'oeuf, tout cela au nom du "respect de l'environnement". A Windsor, près de Sherbrooke, on a construit récemment un gigantesque complexe industriel, une usine de pâtes et papiers de plus de 1,4 milliards de dollars, la plus performante et la plus technologiquement avancée au monde... mais elle rejette tout de même ses déchets liquides dans la Saint-François, une ancienne rivière à saumons... et il semble bien que très peu de gens s"en scandalisent. Par contre, si un propriétaire de chalet va laver ses pinceaux dans l'eau limpide du lac Memphrémagog, il fera l'objet de l'opprobre et de l'indignation populaires. Parce que, voyez vous, les fautes contre l'environnement semblent toujours bien piresquandelles sont d'origine touristique! Que l'industrie touristique doive constamment montrer patte blanche n'est pas nécessairement mauvais car la vraie nature du tourisme n'est pas de détériorer mais plutôt de protéger ou d'améliorer le milieu naturel.

"On doit souligner, en réponse aux accusations selon lesquelles le tourisme porte de graves atteintes à l'environnement, que ce n'est pas le développement du tourisme en soi qui porte en lui les germes d'uneatteinteà $1^{7}$ environnement, mais bien plutôt les méthodes adoptées pour le développement du tourisme en l'absence de responsabilité de la part des pouvoirs publics"(4).

\section{Le tourisme comme outil de protection du milieu}

Le tourisme est à l'heure actuelle un outil privilégié pour contrer les agressions provenant de toutes parts envers le milieu naturel. Dejà en 1970, Jean Dorst ${ }^{(5)}$ estimait que l'aménagement touristique du territoire, pour autant qu'il soit bien compris, devenait un des derniers remparts à la dégradation accélérée et à la destruction systématique du milieu naturel de la planète. L'idée voulant que le tourisme soit un allié essentiel de l'environnement se veut le concept central de notre propos ici car, quand on part en vacances, que recherche-t-on?

"Les atouts principaux du tourisme ne sont pas, comme on 1'imagine souvent, les équipements sportifs et hôteliers, ni les dancings ou autres casinos. Les touristes recherchent au premier chef des sites séduisants, dont le charme et les particularites prennent le pas sur les aménagements touristiques auxquels les promoteurs attachent une telle importance.
Aux yeux des vacanciers, ces équipements ne sont que des moyens pour mieux consommer la nature et le paysage ${ }^{n(6)}$. L'Organisation mondiale du tourisme (OMT) va dans le mềme sens quand elle souligne qu" "une analyse approfondie du comportement des touristes, à partir du moment où ils voyagent pour la première fois jusqu'à l'époque où̀ leurs goûts et leurs aspirations deviennent plus raffinés, démontre que la qualité du produit devient le principal souci du touriste à la recherche progressive du véritable motif de ses déplacements et qui exige une offre plus personnalisée et, par là, une qualité supérieure de l'environnement"(()).

Cette situation est relativement facile à comprendre. Les populations touristiques des pays industrialisés ont maintenant des attentesde plus en plus sophistiquées. Leur niveau d'éducation et les ressources financières qu'elles peuvent consacrer à leurs vacances les incitent à ne rechercher que les produits d'une qualitéc certaine. Cette clientèle est particulièrement sensible et ouverte aux valeurs du milieu naturel(s). Le philosophe amateur tentera donc le sophisme suivant: le tourisme protège l'environnement parce que les touristes recherchent un environnement sain. Les pouvoirs publics veulent recevoir chez eux les touristes à cause des dollars qu"ils apportent. Les pouvoirs publics vont donc se convertir au respect du milieu pour s'approprier les dollars tantconvoités! C'est limpide, non?

"Ainsi, la préservation de l'environnement devient-elle une de leurs préoccupations majeures. Désormais, on recherche une meilleure intégration du tourisme en milieu local en même temps qu'une meilleure rentabilité, ce quise traduit par une tendance à la concentration des hébergements et des équipements touristiques au profit du maintien des espaces verts, une réduction des nuisances et de la pollution, une amélioration de la qualité architecturale..." ${ }^{\text {(9) }}$

Il ne s'agit pas ici de souhaits pieux. Il doit être très clair qu' un environnement détếrioré fera fuir les touristes. Les nombreux exemples relevés par l'OMT sont tout à fait probants à cet égard, mais respect du milieu naturel ne signifie pas interdiction d'agir.

Dans ce domaine comme dans d'autres, il faut éviter de passer d'un extrême à l'autre. Il $n$ 'est pas rare de voir autour de nous des gens qui hier encore prêchaient les vertus de la croissance tout azimut se convertir soudain en "granola grand-prêtre de la croissance zéro" ${ }^{\text {s" C }}$ 'estoublier que la nature est vivante, donc dynamique, mouvante, capable d'adaptation; elle ne saurait être un musée statique, immobile, interdit. La sagesse se trouve plutốt dans l'harmonie, dans ce que le Club de Rome appelle "une croissance dans l'équilibre" ${ }^{p}$. C'est seulementainsiqu'il sera possible d'assurer 1 'inaliénabilité des ressources naturelles pour le bénéfice des générations futures tout en répondant aux besoins de nos contemporains.

\section{Le tourisme pour améliorer le milieu: exemples pratiques}

${ }^{24} \mathrm{La}$ protection, la mise en valeur et l'amélioration des différents éléments constituant l'environnement de l'homme sont parmi les conditions fondamentales du développement harmonieux du tourisme. De même, une gestion raisonnable du tourisme peut contribuer dans une large mesure à protéger et à mettre en valeur l'environnement physique et le patrimoine culturel ainsi qu’à améliorer la qualité de vie de l'homme." (1)

On a vu que le tourisme mal conçu et mal planifié pouvait être une source plus ou moins importante de dégradation des écosystèmes concernés. On a vu aussi que les exigences de la pratique de l'activité touristique sont un puissant levier de conscientisation au respect des ressources d'un milieu. On peut aller encore un peu plus loin en admettant que le tourisme peut contribuer à améliorer le milieu.

Mentionnons, à titre d'exemple, le cas du Parc de la Gorgé de Coaticook, situé à une dizaine de kilomètres au sud de Sherbrooke. Pendant quelques millions d'années, le Coaticook s'est creusé un lit de plusieurs dizaines de mètres de profondeur dans la roche tendre et friable du secteur. Le débit de l'eau y ayant beaucoup diminué au cours des derniers millénaires, ce site ne semblait présenter aucun intérễt, si bien que certains agriculteurs ne virent pas d'inconvénient à y déverser les résidus liquides de leur exploitation et les urbains virent que c'était un endroit acceptable pour se débarrasser sans complication de leurs vieux électroménagers, matelas éreintés et drapeaux canadiens. Puis un jour, quelques visionnaires de la ville de Coaticook et de l'Association touristique de l'Estriecrurent qu'il était possible et fort avantageux de mettre en valeur cette gorge à des fins touristiques. En quelques années, ce dépotoir dangereux, nauséabond et délabré̉ est devenu un parc très propre et entretenu, mettanten valeur les ressources géologiques de cet environnement, les aires d'observation et l'interprétation de la flore et de la faune. On a profité de l'occasion pour restaurer d'anciennes usines et un barrage hydro-électrique, en plus d'y présenter des pièces de théâtre et d'y 
développer une gastronomie régionale. Toutes ces interventions heureuses sont imputables au tourisme, à une qualité d'environnement qu'on a voulu améliorer substantiellement pour la rendre acceptable aux touristes... et, l'an dernier, 63000 visiteurs estivaux y sont venus.

L'amếnagement d'une ancienne sablière à Orford se veut un autre bon exemple d'amelioration du milieu après une intervention touristique. Un promoteur de l'endroit entreprit en 1987 la construction d'un complexe hôtelier de 117 chambres et d'une série de condominiums devillégiature sur ce site abandonné. Souvent, les promoteurs hôteliers désirent $s$ implanter dans un milieu naturel de qualité qu'ils détruiront en partie afin de "s'installer". Dans le casqui nous intéresse, le promoteur a développé un projet sur un site théoriquement "perdu" pour ensuite le réaménager et aussi le récupérer.

Que penser aussi du Comité d'hygiène et d'aménagement des rivières Magog et Saint-François (CHARMES)? Ce groupe, créé en 1975 par des citoyens préoccupés par la pollution de la rivière Magog et des principales plages publiques situées à l'intérieur des limites de la ville de Sherbrooke, s'est engagé en 1981 à aménager les berges de la Magog tout en mettant sur pied des programmes d'éducation et de surveillance pour la population qui fréquente ce milieu. ${ }^{(11)}$

L'implication de CHARMES a permis aux citoyens de Sherbrooke et de ses environs de recouvrer 1"usage des plages, en plus de prévenir la dégradation du milieu. CHARMES a sans contredit permis de développer l'attrait touristique de la Magog.

Bien des exemples de cettenature pourraient s'ajouter ici sur des dizaines de pages. Pourquoi tente-t-on de réintroduire le saumon de l'Atlantique dans la SaintFrançois? Pourquoi le comité CHARMES met-il tant d'efforts à assainir l'eau de la Magog? Pourquoi prohibe-t-on la coupe à blanc sur les rives de lacs et de rivières? Des milliers d'interventions sont effectuées dans le milieu pour le protéger, pour le régénếrer ou pour l'améliorer, afin de le rendre compatible aux standards de qualité des vacanciers.

"L'avenir du tourisme dépend beaucoup plus de la sauvegarde du paysage naturel que d'une mise en valeur intensive. Cela signifieque la protection des paysages reste en toute circonstance prioritaire sur les besoins économiques et techniques de l'industrie touristique. (...)
Cequicaractérise le tourismed'aujourd'hui et de demain, c'est 1'aspiration à un changement radical du cadre de vie et, par voie de conséquence, la nécessité d'un paysage intact. On peut prédire sans risque d'erreur que cette motivation ira en se renforçant. Le paysage à lui seul constitue la matière première, la base même du tourisme et la piecce maîtresse de son Economie." (12)

\section{Les énoncés de politique 1990}

A la fin de 1990, les gouvernements du Québecet du Canada ont rendu public leurs Énonoés depolitiqueen matière de tourisme. Ces documents permettent de se faire une bonne idé́e de la place de l'environnement dans les priorités que la classe politique concède à l'industrie touristique aussi bien québécoise que canadienne.

Dans le document fédéral, un chapitre de 59 demi-lignes (equivalent de 30 lignes) porte sur le développement durable, une expression devenue trèsà la mode ces tempsci dans les officines gouvernementales. L'expression développement durable est employée pour décrire les stratégies privilégiées qui permettent la croissance économique dans le respect de l'environnement. Dans le cas de l'industric touristique, le développement durable consiste à développer l'industrie touristique canadienne et la qualité de ses produits en évitant les répercussions nuisibles à l'environnement et, par le fait même, à l'industrie. Un développement ́́quilibré et responsable pourra neutraliser les motifs de réglementation nouvelle de l'utilisation des terres. En l'occurrence, comme c'est souvent le cas, l'autoréglementation est la meilleure façon d'éviter la réglementation officielle." "(13) Cela dit, le document insiste sur l'importance des parcs nationaux au Canada et sur le fait que $1^{\text {"'tindustrie }}$ touristique devrait faire valoir ses impératifs de développement avec autant d'énergie que le autres industries."

Sous le titre de L'amélioration de la qualité de vie, l'énoncé de politique du gouvernement du Québec en matière de tourisme va à peu près dans le mëme sens que le document fédéral pour ce qui est du volet environnemental. "Le secteur touristique devra à l'avenir accorder une attention particulière à la mise en valeur des ressources dans une perspective de développement durable et de respect de l'environnement. Compte tenu des tendances de la demande touristique et des préoccupations des citoyens en la matière, il faut viser à ce que ce développement s'inscrive dans un cadre harmonieux. En effet, plus que toute autre activité économique, le tourisme permet de rendre compatibles le développementéconomique et la protection des ressources naturelles. (14)

Les deux niveaux de gouvernement indiquent donc qu'ils sont sensibilisés à la relation tourisme-environnement-qualité de vie-6conomic. Cette sensibilisation est toutefoisfort timide et son expressiondonne dans le voeu pieux et l'angélisme. Quant à la volonté politique, c'est évidemment beaucoup demander. On peut quand même se réjouir de constater que ces énonós de politique réaffirment clairement que le tourisme est un facteur d'amélioration de la qualité de vie, d'une part, et que tourisme t respect du milieu sont des réalités quí peuvent cohabiter, d'autre part.

\section{Conclusion: étalement ou concentration?}

Ceux et celles qui estiment que le tourisme détériore le milieu naturel ont raison dans bien des cas et cela est inadmissible. Mais il n'est pas dans la nature de cette industrie de saccager le milieu puisqu'elle a pour objet de répondre aux besoins et aux attentes de vacanciers et voyageurs à la recherche d'un cadre naturel sain et propre, d'une eau pure, d'une ambiance calme et reposante. Les agressions contre l'environnement sont donc le fait d'un développement avec des objectifs économiques à court terme d'un manque ou d'une absence de planification, et d'une absence de conscience sociale et environnementale. Car, si on a le respect de son milieuet de laclientèle que l'on souhaite desservir, le tourisme devient un serviteur et un protecteur de l'environnement physique, mais aussi social, culturel et économique. Maintenant que les municipalités régionales de comté (MRC) ont toutes des schémas d'aménagement adoptés et qu'elles disposent de moyens de contrôle très explicites, il devrait être plus facile de s'assurer que le respect du patrimoine collectif a priorités sur celui des pollueurs.

Parmi les moyens qui sont mis de l'avant pour limiter les pressions mises sur l'environnement malgré la croissance continuelle des flux touristiques, il y en a deux quel'on retrouvechez tous les auteurs: la concentration des equipements et l'étalement des vacances.

La problématique de la forte concentration des vacances dans le temps est discutée depuis déjà quelques décennies: l'industrie injecte des dizaines de millions de dollars à chaque année dans des équipements qui bien souvent ne servent que deux ou trois mois parannée. Pour répondre aux attentes des périodes de pointe, on construit 
(suile de la page 5)

beaucoup et on bétonne de vastes espaces. Des vacances étalées sur une base plus large permettraient de repondre aux besoins d'un plus grand nombre de vacanciers avec moins d'équipementseten protégeant donc mieux $l^{*}$ espace naturel de la station. Mais si les touristes avaient plus de latitude pour choisir le moment de leurs vacances, il n'est pas dit qu'ils en feraient un meilleur étalement. Cette question devra être traitée conjointement avec celle des vacances scolaires.

La concentration des équipements récréotouristiques dans l'espace est un sujet plus controversé. Les auteurs consultés estiment qu'un bon moyen de protéger les milieux naturels fragiles en zone touristique est de concentrer les équipementset services. Par exemple, un hôtel de centchambresconstruit sur un terrain de deux acres agressera moins l'environnement que cent résidences secondaires construites sur les rives d'un lac sur des terrains "aménagés" d'un acre chacun. Mais ce ne sont pas tous les aménagistes, ni tous les urbanistes, ni tous les ćcologistes qui partagent cette approche, qui $n^{7}$ estd'ailleurs pas sans nous faire penser à des politiques gouvernementales ou à des approches de devveloppement discutées sérieusement à haut niveau. Par exemple, quand on a institué, il y a une quinzaine d'années, le programme du Crédit touristique, on avait limité son application à des portions de territoire bien délimitées en fonction de la qualité du potentiel touristique sur les plans régional, national ou international, La loi 37 identifiait donc des portions de territoire comme station touristique, comme pôle touristique, comme corridor touristique, etc, et les ressources du Crédit touristique n'étaient admissibles qu'à 1'intérieur de ces espaces. Voilà un moyen de concentrer et d'intégrer les différents produits touristiques... et sans doute de protéger l'environnement.

Plus près de nous, à l'arrivée en fonction du ministre André Vallerand et de son sousministre Michel Roy, on avait commencé à aviser les Associations Touristiques Régionales (ATR) que $\mathrm{l}^{\text {*intention }}$ gouvernementalétaitd d'appuyer davantage les régions disposant d'importantes ressources récréo-touristiques tout en réduisant l'appui aux régions moins bien pourvues. On a laissé tomber cette approche parce qu'elle n'était pas "électoralement rentable". Car, au pays du Québec, c"est bien souvent la rentabilité politique qui détermine ce qui est bien et ce qui est mal. Et cela n'est pas propre au Québec...

\section{Notes explicatives}

11) KFIPPENDORF, Jost (1977), Les dévoreurs de paysages - Le tourisme doit-il détruire lessites quil le font viure? Editions 24 heures, Lausanne. 1. 88

(2) ROY, Jean-Hugues (1990), "Pas devacances pour Femironnement - La tourista ronge la planete". revue Écologie, Montréal, p. 8

3) KRIPPENDOFF Jost 11977 , lbid, D. 56

4) OMT (1980) Etude sur la contribution du tourisme a la protection de l'environnement. Rapport del'Assemblese genéraledel'Organisation mondiale du tourisme, New Delhi, pp. 10-11

(5) DORST, Jean (1970). La nature denaturée. Editions Delachaux \& Niestle, Collection "Points", no 9, 192 pages.

(6) KRIPPENDORF, Jost (1977), lbid, p. 17

(7) OMT (1993) lbid p. 10

(B) NADEAU, Roger (1963), "Le milieu naturel québeoois, "e'est beau engrand" ", revue Téoros, wol. 2, no 2, UQAM, Montreal. UApouyé par I'ouvrage de KALAORA, Bernard (1981), Le musée vert- oule tourisme en forät, Editions Anthropos, Paris.

(9) OCDE (1980). L'Impact du tourisme sur Fenvironnement. Rapport géneral de IOrganisation de cooperationet dedeveloppement economique, Paris, p. 41

(10) OMT (19B3), lbid, p. 2

(11) NADEAU, Roger (1909), "Le recreo-tounisme en Estrie: un apport positif à la gestion de "environnement", Le recréo-tourisme: une nouvalle voie dé dóvaloppement. Association professioninelle des géographes du Quebec APGGOI at Module de gégraphie de l'Universite du Québec à Chicoutimi, sous la direction de JuanLuis Kein et Bemard Maltais, Chicoutimi, pp. 123 134

(12) KAIPPENDORF, Jest 11977), Ibid, PP. 90 et 47

(13) CANADA (1990), Le tourisme, un pas à franchir, Industrie, Sciences et Technologie Canada. Ottawa, pp. 21-22

114] Quebec (1990). Enonoé de politique en matlère de tourisme. Document de réflexion. Gowvernement du Quebec, ministere du Tourisme. Québec, p. 40

(suite de la page 25)

pressure of other problems related to the city, e.g., education, saving local art groups such as the symphony, fiscal reform and the March 3 rd tax proposal.

Another way of analyzing the JCT's situation is to view it as a conjugate collective (Astley and Fombrun, 1983). Such collectives are those in which members from different sectors of the same industry (e.g., preservationists, and tourism-related firms) are related to each other, respectively, in term of their inputs and outputs (Evan, 1966). Interindustry collaboration in conjugate collections relies quite a bit on legal sanction and formal agreement as control mechanisms (Astley and Fombrun, 1983). In the case of the JCT there were no such control mechanisms. Of the two universities involved, one hesitated to sign an agreement reflecting the joint partnership of these two universities in the JCT.

In summary at this point, the progress of the JCT has primarily been thwarted by a lack of resources and formalization of an agreement. On the other hand, a subgroup of preservation-oriented individuals has evolved.

\section{Formation of the Cultural Treasures Subgroup}

An interesting spinoff of the JCT is the formation of another coalition as described above. Although it is too early to evaluate the subgroup's success in achieving its objectives, several observations are in order. For one, the membership reflects a strong interest in preserving the Vieux Carre, improving site (museum) and event visitations. Two of the key leaders are strong preservationists. Collectivelly, this group desires to create a stronger force in dealing with the City of New Orleans governmentso problems of the Vieux Carre such as litter, the homeless, and crime can be addressed. This would make the Vieux Carre a more livable place for residents and an interesting attraction for tourists. In contrast to the JCT, the subgroup is more cohesive. Members possess similarvalues, especially in preserving the Vieux Carre. On the other hand, the spinoff group has a problem similar to that of the JCT, a lack of resources, especially money.

A review of the cooperative marketing strategiesdeveloped by this group (see Table 3) suggests several concerns. As in the case of the JCT, all members are on a volunteer basis. Secondly, many of the marketing effortse.g, signage, window displays, tradeshow displays, video production, and brochures, will cost money unless tradeouts can be arranged with suppliers of such services. Lastly, no provision is being made for a full-time director, one who can coordinate the efforts of coalition members.

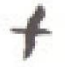

Bibliography

ASTLEY, W.G. and FOMBRUN, C.J. (1983), Collective Strategy: Social Ecology of Organizational Environments, Acadomy of Mana gement Review, B (4), $576-587$

BAUMBACH, Ir. R.O., BROOKS, J.S., ELSTROTT, Ir J.B. and A.M. MASSON (1989), Issuas and Opportunities in Cultural Tourism, Unpublished report to the National Trust for Historic Preservation

coOTE, EA. (1982). The Vieux Carre: 1961 to 1981 . New Orleans, LA, Loyola University, College of Business Administration.

CYERT, R,M and MARCH, J G. [1963). A Behavioral Theory of the Firm, Englewood Cliffs, NJ. PrenticeHall Ind

EVAN W (1966). The Organizations - SeL Toward a Theory of Interorganizational Relations, in $J . \mathrm{D}$ THOMPSON led.I. Approaches to Organizational Design, Pittsburgh. University of Pinsurgh Press, 175-190.

HUNT, J.D. (1990), State Tourism Ofrices and theit Impact on Tourst Expenditures, Jeurnal of Travel Research, 25 (3), 10-13

INSKEEP, E. (1968). Towism Planning An Emerging Specialization. Journal of the American Planning Association, 54 6937, 360-372

KATZ,A. (1990, January 151. How New Oreans Achieved a Reputwion? as a Big Event Town, City Business, pp $21-23$

MURPHY, P.E. (1985), Tourism: A Community Approach. New York, NY, Wethuen Inc.

NEWMAN, WH LOGAN, IP and WH HEGARTY [1989). Strategy: A Multi-Level Integrative Approach, Cincinatt, $\mathrm{OH}_{4}$ South-Westem Publishing Co.

NIELSEN, R.P. (1988), Cooperative Strategy, Strategic Management Journal, 9, pp. 475-492

PECKHAM, PG 11989. Cultural Tourism in New Orleans: A Case Study of Tourism Planning and Management Strategies, unpublished Master's thesis, University of New Orleans, New Orleans, LA. RODDEWIG, R.J. (1980), Selling Amenica's Heritage. Whout Selling Out, Preservation Forum, 2 (3), pp 2-7

WINANS, C. (October 3. 1989). For U.5. Tourism the Holiday may be Over. Wall Street Journal, B 16.

WAGNER, F and NEBEL, E (1984), Facing Change In the Vleux Carro, New Orleans, LA, University of New Orleans, School of Urban and Regional Studies and School of Hotel, Restaurant and Tourism Maracement 